\title{
Dynamometry testing in spinal cord injury
}

\author{
Sue Ann Sisto, PT, MA, PhD; ${ }^{1-3^{*}}$ Trevor Dyson-Hudson, MD $^{1,4}$ \\ ${ }^{1}$ Department of Physical Medicine and Rehabilitation, University of Medicine and Dentistry of New Jersey, New Jersey \\ Medical School, Newark, NJ; ${ }^{2}$ Department of Physical Therapy, School of Health Related Professions, Newark, NJ; \\ ${ }^{3}$ Human Performance and Movement Analysis Laboratory, Kessler Medical Rehabilitation Research and Education \\ Center (KMRREC), West Orange, $N J ;{ }^{4}$ Spinal Cord Injury Laboratory, KMRREC, West Orange, NJ
}

\begin{abstract}
Persons with a spinal cord injury (SCI) demonstrate strength deficits that can limit their functional ability to perform activities of daily living. For a specific lesion level, performance of functional activities is related to the level of muscle strength. Consequently, in clinical practice, we need reliable measures of muscle strength to determine mobility and selfcare ability. Muscle-strength testing is used to document recovery or loss of motor function early in SCI, as well as measure improvements in strength in chronic SCI. We also need such measures for research purposes to determine the efficacy of clinical trials. Several methods are available for testing muscle strength of persons with SCI, such as handheld, handgrip, and isokinetic dynamometers. This article provides an overview of muscle-contraction definitions and testing methodologies and discusses the reliability of these testing methods and dynamometry devices.
\end{abstract}

Key words: dynamometry, functional ability, grip strength, handheld dynamometer, manual muscle test, muscle contraction, muscle strength, rehabilitation, spinal cord injury, strength deficit.

\section{INTRODUCTION}

Persons with a spinal cord injury (SCI) have strength deficits that can limit their functional ability to perform activities of daily living (ADL). For a specific lesion level, performance of functional activities is related to the level of muscle strength, particularly in subjects with tetraplegia [1]. For example, upper-limb muscles must be strong enough to support the body for pressure relief and transfers. Wrist muscle strength has been related to the motor component of the Functional Independence Measure (FIM) and self-care scores, and therefore, wrist strength is important in ADL [2]. In clinical practice, we need reliable measures of muscle strength to determine mobility and self-care ability. Muscle-strength testing is used to document recovery or loss of motor function early in SCI, as well as measure improvement in strength in chronic SCI. Occasionally, posttraumatic syringomyelia is present, in which motor and sensory function can deteriorate and an accurate method for reassessment of muscle strength is needed. In the case of spinal cord disease or dysfunction that may be progressive, such as multiple sclerosis, close monitoring of muscle-strength changes is warranted. We also need such measures for research purposes to determine the efficacy of clinical trials, such as functional ability changes resulting from exercise or other interventions.

\footnotetext{
Abbreviations: ADL $=$ activities of daily living, ASHT = American Society of Hand Therapists, ASIA = American Spinal Injury Association, FIM = Functional Independence Measure, $\mathrm{HHD}=$ handheld dynamometer, ICC = intraclass correlation coefficient, $\mathrm{MMT}=$ manual muscle testing, $\mathrm{ROM}=$ range of motion, SCI = spinal cord injury.

* Address all correspondence to Sue Ann Sisto, PT, MA, PhD; Human Performance and Movement Analysis Laboratory, KMRREC, 1199 Pleasant Valley Way, West Orange, NJ 07052; 973-243-6888; fax: 973-234-6984.

Email: ssisto@kmrrec.org

DOI: 10.1682/JRRD.2005.11.0172
} 
Several methods are available for testing muscle strength of persons with SCI. These include manual muscle testing (MMT) and dynamometry. Dynamometry includes the use of handheld dynamometers (HHDs), handgrip dynamometers, and isokinetic dynamometers. This article provides an overview of muscle-contraction definitions and strength-testing methodologies and discusses the reliability of these testing methods. Additionally, the reliability of specific dynamometers is discussed.

A review of the definition of muscle-contraction parameters may be useful for understanding musclestrength testing methodologies. Strength is the force or moment produced by a single maximum voluntary isometric contraction. Strength refers to the ability of a muscle to develop active tension that produces force. Muscle power is the rate of doing work and, therefore, the product of force and velocity. Functionally, muscle power is assessed by dynamometers that dynamically measure force, while simultaneously measuring or controlling the movement velocity, such as with isokinetic dynamometers.

When examining muscle strength using dynamometers, we must consider two relationships. The first is the length-tension relationship of the muscle. This relationship is between the maximum tension in a muscle versus its length. Ideally, the muscle strength should be tested in its optimal length-tension position. For example, to measure the strength of the biceps, we should position the elbow at approximately $90^{\circ}$, which would put the muscle at its optimal length and generate the greatest tension. However, since the biceps is a two-joint muscle, standardizing the position of the shoulder is important as well. Persons with SCI may have difficulty assuming certain positions and therefore testing their muscles in the optimal length-tension position may be difficult. The greatest challenge for standardization of testing position is gravity. When a gravity-eliminated position is needed for testing, a person with SCI may be unable to assume such a position while still maintaining the optimal lengthtension in the muscle being tested. For example, when shoulder-abduction strength is being tested in the supine gravity-eliminated position, the person needs to maintain the shoulder in neutral rotation but then may not have sufficient shoulder-rotation strength for the shoulderabduction testing. In this case, notations should be made about limb stabilization and any alternative testing positions.

The second relationship is the force-velocity relationship. This relationship is between the maximum muscle tension and velocity. Maximum force is inversely related to the velocity of muscle shortening. Isokinetic dynamometry training takes advantage of this relationship by creating greater torque and power output at training velocities [3]. When an HHD is used, different rates of force application against the dynamometer will produce variable force production. Standardizing the time expected to get to a maximum contraction may reduce this variability.

Understanding the definitions of the types of muscle contractions is also important for strength testing. The types of muscle contractions are isometric, isotonic, and isokinetic. Isometric muscle contractions are those that have a constant length or static contraction with a zero velocity of shortening. Isotonic muscle contractions involve movement under a constant load throughout the range of motion (ROM), such as when a weight is lifted. Isokinetic contractions are those in which a load is moved at a constant rate of muscle contraction throughout the ROM. Most HHDs measure the isometric type of contraction, and isokinetic dynamometers can test the strength of both concentric and eccentric contractions. Concentric contractions cause muscle shortening, while eccentric contractions cause muscle lengthening.

Finally, when performing MMT or using an HHD, we commonly use two types of tests. These are defined as the "make" and the "break" tests. For the make test, the examiner applies resistance in a fixed position and the person being tested exerts a maximum effort against the dynamometer and examiner. The examiner is only required to sustain an isometric contraction and read the highest value on an HHD, if one is used. For the break test, the examiner applies resistance sufficient to overcome the maximum effort of the person being tested and cause the subject's joint to move in the opposite direction, which results in an eccentric contraction [4]. The break test requires that the examiner have additional skills, such as the ability to move the limb at a constant velocity while the person being tested is asked to achieve a maximum contraction. In addition, if an HHD is used, the examiner must read the force output reading at the exact time the subject's limb begins to move. For example, a break test for shoulder abduction requires the examiner to apply resistance that overcomes the subject's shoulder abductors at the distal end of the humerus such that the arm begins to move back into adduction. The instructions for the break test are for the examiner to apply as much force as possible without allowing any movement from the starting angle of the joint. The examiner must exert sufficient counterforce to overcome the maximal force exerted by the person being tested. Conversely, the instructions 
for the make test are simply to have the person being tested apply as much force possible against a fixed resistance. However, because the two tests measure different forces, they cannot be used interchangeably [5]. Bohannon reported significantly higher strength values for the break test; however, the tests were performed on the elbow flexors of nondisabled females [6]. This finding was replicated in a subsequent study that evaluated the paretic and nonparetic limbs of persons with stroke [4].

In deciding whether to use the break or make test approaches, we must understand the reliability of each test. Previous studies are not in agreement; break tests were found to be more reliable than make tests by 10 to 70 percent [5,7]. Bohannon reported that the interclass correlation coefficients (ICCs) were similar when the elbow flexors of nondisabled females were tested (0.91 and 0.92 for the make test and break test, respectively) [6]. Bohannon compared make and break tests of the paretic and nonparetic limbs of patients with stroke and found that the break tests resulted in significantly higher strength measurements, particularly for the paretic limb. The author concluded that although make and break test measurements were reliable (ICCs were 0.98 and 0.97 for the make and break tests, respectively), the forces produced within and between the paretic and nonparetic limbs were different. Bohannon also concluded that the two tests should not be used interchangeably and recommended the break test when the influence of excess muscle tone is to be reduced [4].

Others have found slightly greater differences in the reliability of the break test and attribute it to the additional skill required by the examiner [5]. However, Burns et al. compared make and break tests of the elbow flexors of persons with tetraplegia that were performed by two inexperienced examiners [8]. Electrogoniometric measures indicated that the examiners performed the tests in a similar manner, and the minor differences in technique were not associated with the differences in strength measured. The authors found excellent inter- and intrarater reliability for the HHD used in the tests and concluded that no clear reason for choosing one method over the other existed. The variation between earlier studies and that of Burns et al. may be attributed to differences in the strength of the muscles being tested Further research is needed to replicate these findings for strong and weak muscles of persons with SCI.

\section{MANUAL MUSCLE TESTING}

The American Spinal Injury Association (ASIA) [9], in their International Standards for Neurological Classification of SCI, recommends muscle-strength testing with MMT. MMT is the most widely used clinical tool for strength testing because it can be performed quickly, understood easily, and reproduced when severe weakness is measured [10]. MMT scores muscle strength according to whether the muscle can move the lever arm against gravity (3/5-5/5), without gravity (2/5), or demonstrate a palpable contraction (1/5). However, MMT has been reported to be relatively insensitive to changes in muscle strength because it depends on the examiner's judgment of the amount of resistance applied during the test [10]. This judgment can be especially difficult depending on differences in subjects' preinjury physical fitness, age, and sex [11]. The experience of the examiner can influence the consistency of MMT scores [12]. Furthermore, the range of certain MMT grades, such as grade 4, is too broad, which makes detecting strength changes difficult.

Standardized positions are used to perform MMT for each muscle group [13]. MMT takes a considerable amount of skill to master and still ensure inter- and intrarater reliability [14]. However, subtle changes in muscle strength may be obscured when MMT is used [10]. For example, several researchers have reported that strength of grade 4 on the Medical Research Council scale of 0 to 5 cannot predict strength and may represent only 10 percent of the predicted strength [15-16]. When using MMT, the examiner rates muscle strength on an ordinal scale that has limited reliability and responsiveness, particularly for grades $3 / 5$ and greater; whereas, when dynamometry is used, strength is measured on a continuous scale. This continuous measurement allows for greater accuracy and is more suitable for reliable measures of strength [17].

\section{DYNAMOMETRY TESTING PROCEDURES}

In general, instrumented strength testing has been demonstrated to be more reliable than MMT [18]. Dynamometers are devices that measure force or power. Several classes of dynamometers are available to the clinician and researcher for measuring the strength of persons with SCI. These include HHDs, handgrip dynamometers, and isokinetic dynamometers. 
A standard testing protocol and position is important for reliability and comparison of normative data. The test protocol should include a description of the testing position and the instructions used [19]. A decision must be made on the number of trials (usually three) and whether the best or the average force values will be used. The examiner must decide if encouragement is provided and if so, ensure that the encouragement or coaching is consistent across all testing periods. The rest period in between trials should be established. Generally, rest periods range from 15-60 s so that declines in strength across trials due to fatigue are avoided. The duration of isometric trials should also be standardized so that a maximum reading can be obtained. Generally, the duration ranges from 3-5 s. For isotonic contractions, the rate of contraction or duration of contraction should be standardized so that responses based on variable times for motor unit recruitment are avoided. For isokinetic testing, the duration of contraction is established by the preset speed of the dynamometer; however, instructions and coaching should be provided to elicit a consistent effort. For example, subjects should be instructed to contract the muscle as hard and fast as possible. Finally, patient warm-up trials decrease the variability of the strength measurements. Sex, age, and weight are predictors of muscle force on dominant and nondominant sides [20] and should therefore be considered when strength is compared between individuals.

\section{HANDHELD DYNAMOMETER}

HHDs, also known as myometers, have several advantages over other types of dynamometers, including lower cost, greater ease of use, and better acceptability in clinical settings. Researchers and clinicians are evaluating the benefits of HHDs for strength measurement in persons with SCI. If a device is used for strength measurements, it should be reliable, portable, sensitive to change, and easy to use [21]. The portability of these devices enables the testing of multiple muscles over a short time period and allows for bedside testing. HHDs are quite affordable and range from $\$ 400$ to $\$ 1,000$. Despite encouraging reliability studies, concerns have been raised about stabilizing the dynamometer and subject during the test. HHDs are superior to MMT for detection of mild-tomoderate changes in muscle strength [10].

Disadvantages of the HHDs include that they are capable of measuring only one point in the ROM at a time, that the examiner may be overcome by the subject's power, and that the examiner may provide inconsistent counterresistance during strength testing. Therefore, when using an HHD, the examiner must be able to provide appropriate stabilization and the examiner's strength must be greater than that of the muscle group being tested. Some researchers have suggested that HHDs be used to test persons with weaknesses rather than the nondisabled [22]. Although persons with SCI have both weak and strong muscles, the HHD testing procedures will be more variable for the strong muscles if the examiner is unable to provide adequate stabilization and resistance. Stability and resistance are more easily provided when persons with SCI are tested than they are with competitive athletes. Since many tests of subjects with SCI are performed while they are seated, the examiner can position him- or herself to provide adequate resistance.

HHD testing can also be performed at several points in the ROM, if necessary for interpreting functional movements; however, the optimal length-tension relationship may be lost in certain positions and these positions may not be easily repeated or standardized for follow-up tests. When using an HHD for strength testing, the examiner must determine the testing point in the ROM based on the functional demands of the subject. For example, strength testing for functional wheelchair use may require the examiner to position the subject at or below $90^{\circ}$ of shoulder flexion, rotation, and abduction, whereas testing for overhead reaching tasks performed during ADL may require the subject to be positioned above that level. However, the examiner must weigh the emphasis of testing in a functional position against taking advantage of the length-tension relationship of the muscle group tested. Above all, the position in the ROM identified for strength testing must be standardized for all measurement intervals for a given patient. Otherwise, comparisons of change in strength over time will result in spurious interpretations. Furthermore, performing isometric testing at multiple points in the ROM is not the same as an isotonic or isokinetic dynamic contraction.

HHDs that have been used for strength testing include, but are not limited to, Isobex (Curson AG, Bern, Switzerland), Chatillon ${ }^{\circledR}$ MSC Series dynamometer (AMETEK, Inc, Largo, Florida) (Figure 1), Lafayette Manual Muscle

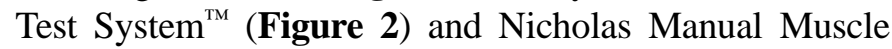
Tester $^{\mathrm{TM}}$ (Figure 3) (Lafayette Instrument Company, Lafayette, Indiana), and microFET2 ${ }^{\mathrm{TM}}$ (Figure 4) (Hoggan Health Industries, Inc, West Jordan, Utah). The Isobex dynamometer is a microprocessor-controlled device that 


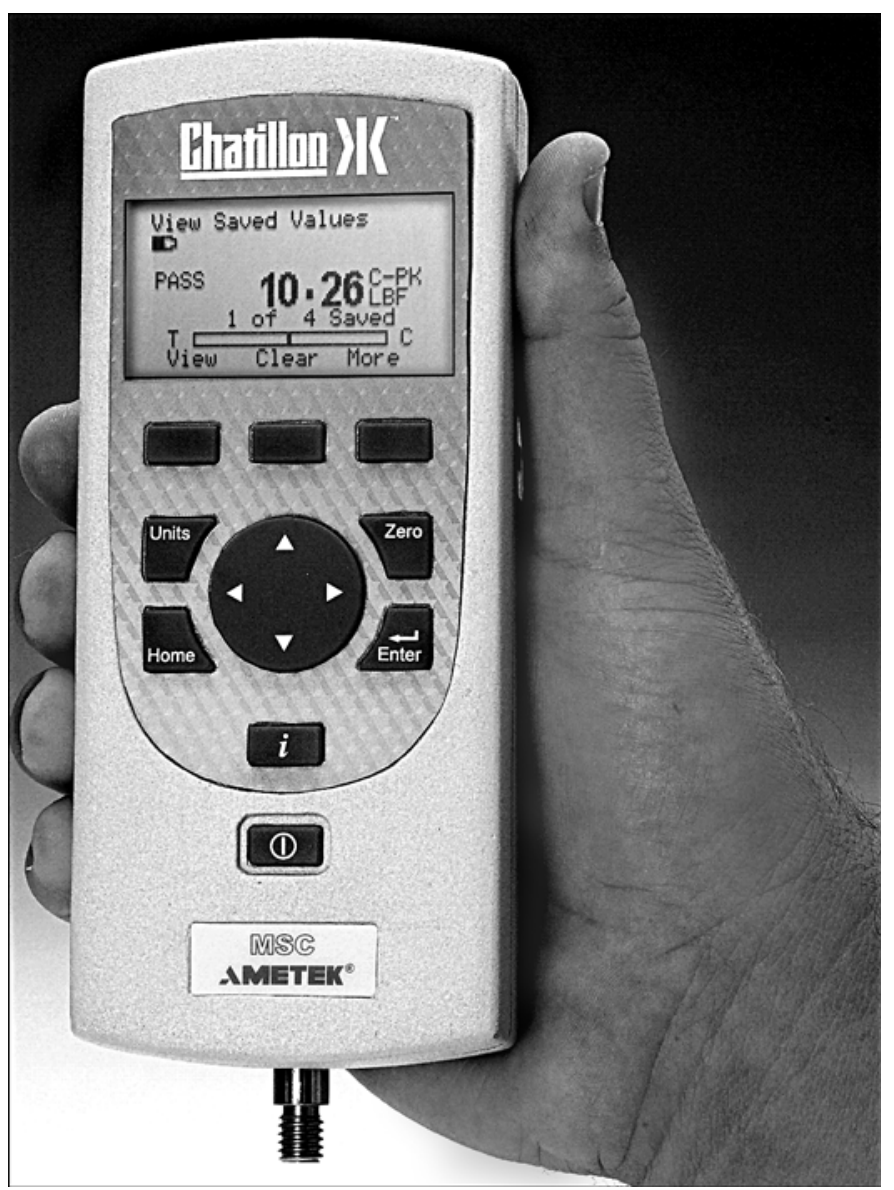

Figure 1.

Chatillon ${ }^{\circledR}$ MSC Series dynamometer (AMETEK, Inc, Largo, Florida). Used to test muscle strength and has "U"-shaped attachment (not shown) that provides comfortable contour when significant pressure is applied to limb segment. Figure courtesy of AMETEK, Inc.

has a $1 \mathrm{~kg}$ minimum threshold. It disregards the first second of data because force output rapidly increases in the initial period [23]. The sampling rate is adjustable so that the number of samples is optimized depending on the speed of contraction.

The Chatillon MSC Series dynamometer measurement accuracy is $>0.1$ percent full scale; a liquid-crystal display supports a variety of measurements, including normal and peak readings, dominant versus nondominant comparisons, pass-fail results, statistical results, load averaging, load comparisons, measurement actuation, and direction. The display can be inverted, and displayed results may be hidden from the subject during testing. This device collects a continuous analog signal that can be downloaded to a computer or viewed on the digital screen.

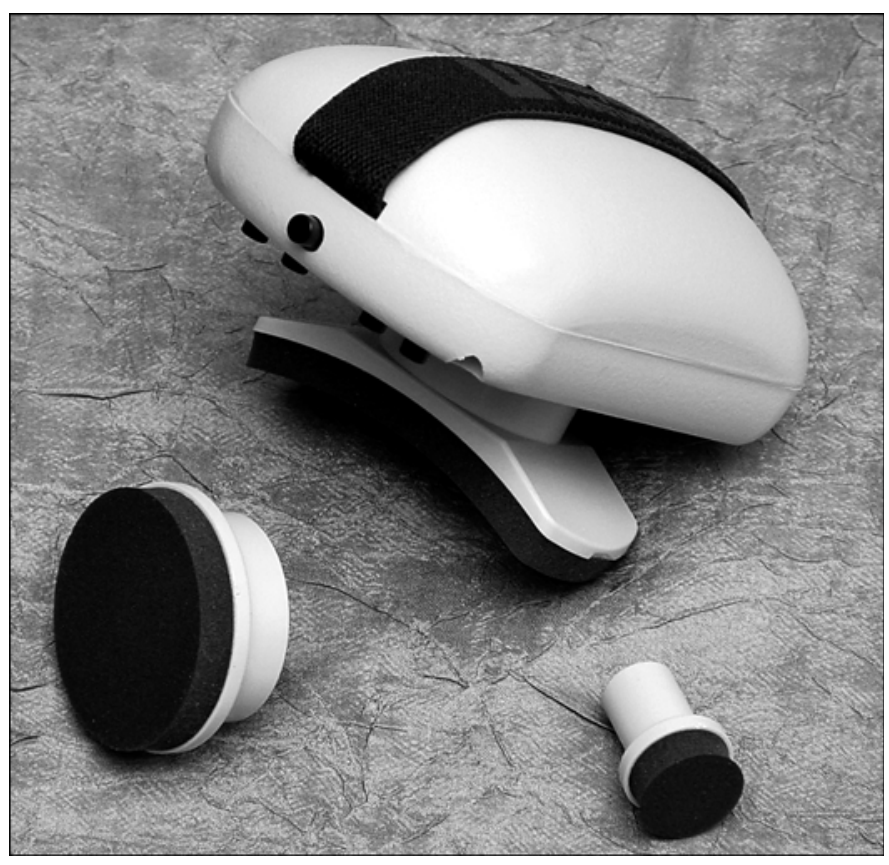

Figure 2.

Lafayette Manual Muscle Test System ${ }^{\mathrm{TM}}$ (Model 01163) (Lafayette Instrument Company, Lafayette, Indiana). Lafayette Manual Muscle Test System provides comfortable grip against which counterresistance is provided. Curved attachment provides best comfort on contoured surface. Other attachments provide more directed force for smaller surface areas. Figure courtesy of Lafayette Instrument Company.

The Lafayette Manual Muscle Test System features a lightweight (10.6 oz) microprocessor-control unit that measures peak force (pounds or kilograms), time to reach peak force, and total test time, while storing up to 52 tests. Test times can range from 1-10 s, and an audible tone indicates the end of the preset time. The unit provides a built-in calibration routine that verifies a valid calibration. The software menus allow browsing of device options. It has an accuracy of \pm 1 percent, and the battery life is $85 \mathrm{~h}$.

The Nicholas Manual Muscle Tester was developed by the orthopedic surgeon James A. Nicholas, MD, of the Nicholas Institute of Sports Medicine and Athletic Trauma at Lenox Hill Hospital in New York City. It contains a patented load cell that minimizes errors from nonperpendicular loading. The Nicholas Manual Muscle Tester can measure force to $0.1 \mathrm{~kg}$, with a range of $0.9-$ $199.9 \mathrm{~kg}$. It is ergonomically designed to support the tester's hand and has a visible window for reading force output during a break test. This device is no longer manufactured, although many clinics may still use it. The 


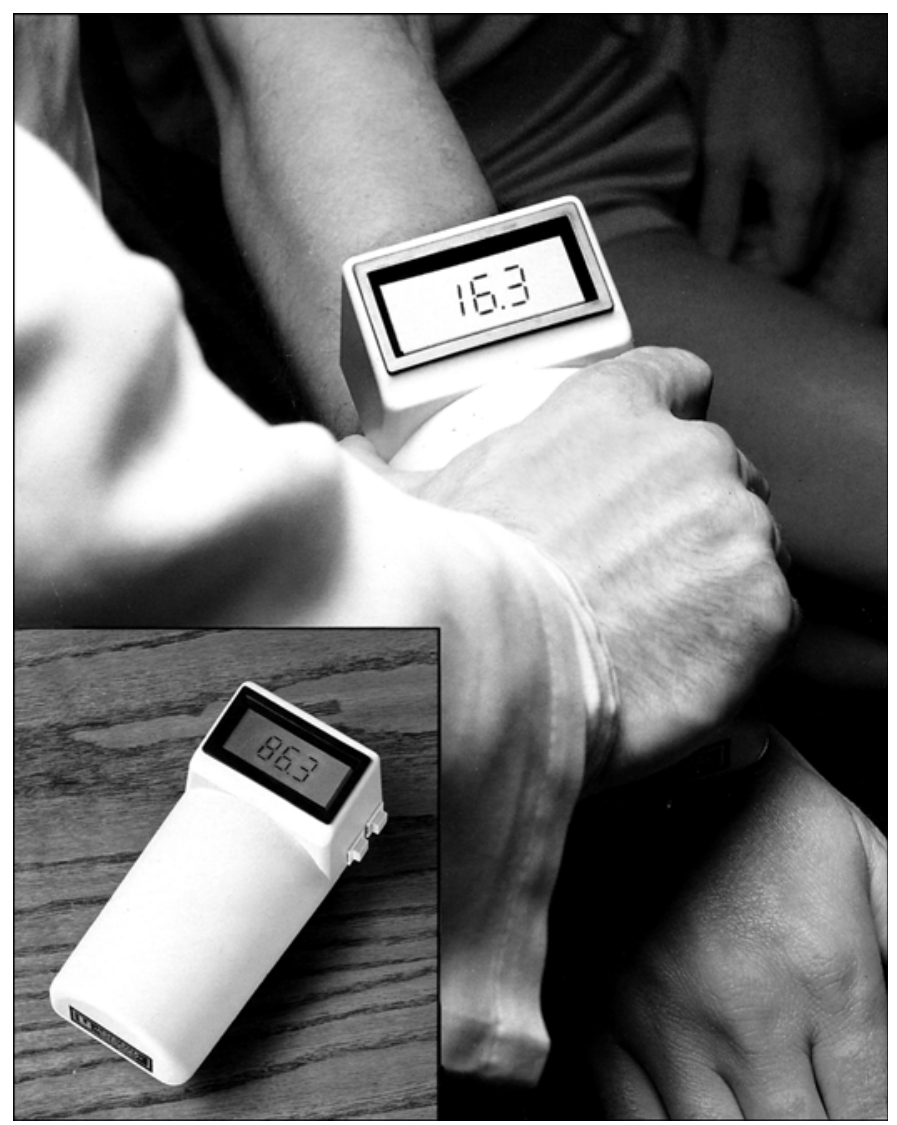

Figure 3.

Nicholas Manual Muscle Tester ${ }^{\mathrm{TM}}$ (Lafayette Instrument Company, Lafayette, Indiana) has been discontinued and replaced with Lafayette Manual Muscle Test System (Model 01163) (see Figure 2). Nicholas Manual Muscle Tester continues to provide excellent ergonomic comfort for examiner, while enabling force output reading without removal of dynamometer from subject's limb. Figure courtesy of Lafayette Instrument Company.

Nicholas Manual Muscle Tester has been replaced with the new version of the Lafayette Manual Muscle Test System seen in Figure 2. The Nicholas Manual Muscle Tester, however, has a desirable feature that allows the examiner to read the force output without removing it from the subject's limb.

The microFET2 muscle strength testing system is approved by the U.S. Food and Drug Administration and has an accuracy of \pm 2 percent. The load cell has a capacity of $150 \mathrm{lb}$ and low- and high-threshold settings that provide dual sensitivity for the measurement of trace changes. The device comes with three pads that match the body segment being tested and fits easily into the palm of the hand. The device is quite durable and suitable for a busy clinical

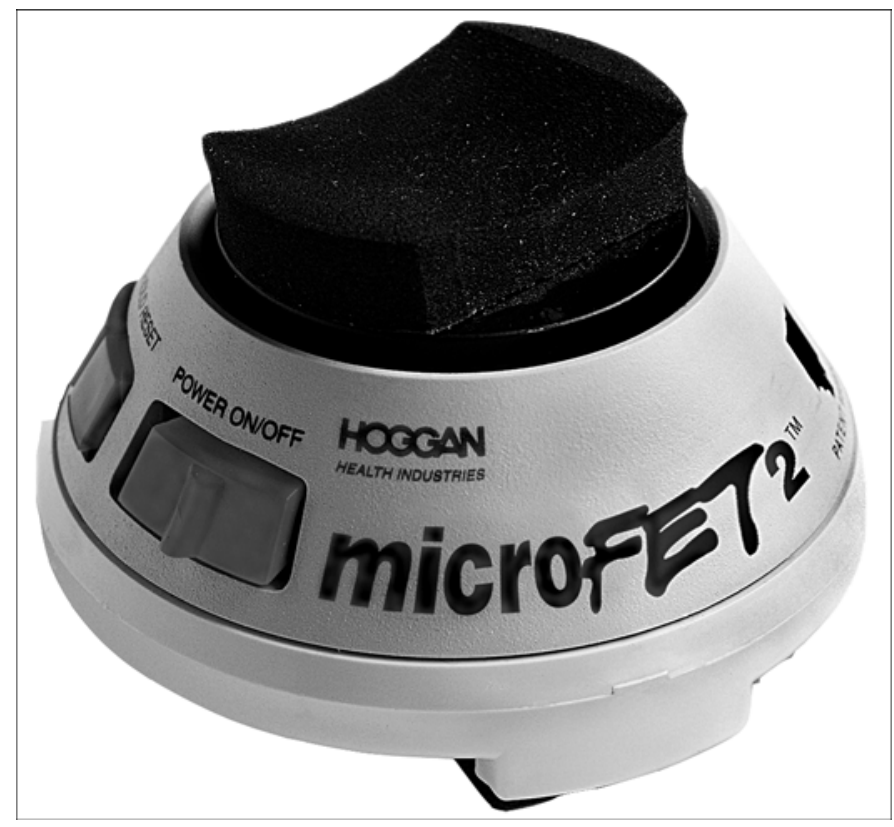

Figure 4.

microFET2 ${ }^{\mathrm{TM}}$ muscle strength testing system (Hoggan Health Industries, Inc, West Jordan, Utah). microFET2 muscle strength testing system fits compactly in palm of examiner's hand and enables scaling adjustment for testing very weak muscles. Cup contour increases subject comfort. Figure courtesy of Hoggan Health Industries, Inc.

practice. Leggin et al. evaluated the strength testing time requirements and found that the shortest amount of time was required for the Nicholas Manual Muscle Tester, followed by the Isobex, and then the Biodex Isokinetic Dynamometer $^{\mathrm{TM}}$ (Biodex Medical Systems, Inc, Shirley, New York) [24].

When strength testing is performed with an HHD, guidelines for optimal repeatability should be followed. The examiner should perform three trials and allow the subject adequate rest between trials. Repeating trials allows the examiner to take an average that will be more representative of a maximum effort. Additionally, the examiner can eliminate outliers or extremely high or low values caused by a subject's inconsistent effort or an examiner's inadequate resistance. The examiner needs to stabilize him- or herself so that the counterforce is in the required direction. The examiner should avoid providing counterresistance across two joints and must pay attention to strategies a patient may use to compensate for muscle weakness. When such compensation is observed, the examiner should terminate the test and the patient should be instructed to avoid compensatory maneuvers. 
When a break test is performed with an HHD, the extent to which the examiner holds the dynamometer at an angle perpendicular to the subject's limb will also influence the measured force. Measurements outside the perpendicular angle will lead to spurious increases in force measurements by adding another force component to the normal force applied [5].

Upper-limb muscle strength of persons with SCI is often tested. Maximum isometric strength testing with MMT and a dynamometer have been described for the shoulder muscle groups, including the shoulder flexors/ extensors, abductors/adductors, internal/external rotators, and elbow flexors/extensors [25-27]. The shoulder flexor/extensors are generally tested with the shoulder and elbow flexed at $90^{\circ}$ and the arm in a neutral rotation, with the resistance or myometer placed proximally to the humeral epicondyle. When testing the shoulder abductors/adductors, the examiner places the subject's arm in $90^{\circ}$ of shoulder abduction (neutral position relative to rotation) and elbow flexion and the resistance or myometer is placed proximally to the humeral epicondyle. The elbow flexors/extensors are tested with the shoulder adducted to the trunk, the elbow flexed to $90^{\circ}$, and the forearm in neutral rotation relative to pronation/supination. The examiner must be positioned properly so safe and consistent counterresistance is applied. Consistency is determined by placement of the counterresistance along the wheelchair lever arm. The examiner should seek assistance for trunk stabilization during testing.

\section{RELIABILITY OF HANDHELD DYNAMOMETERS}

Magnusson and colleagues evaluated both inter- and intraday reliability of an HHD and measured shoulder abductor strength with the break test (which included an eccentric component) [28]. Six maximum trials were collected on 5 consecutive days, separated by 1-2 weeks and excellent reliability was found. Intraday correlations ranged from 0.88 to 0.99 and interday correlations ranged from 0.94 to 0.98 . The authors suggest that measured strength changes of $<11$ percent are within measurement error and should not be considered clinically significant.

Burns and Spanier conducted a study of the break and make tests using an HHD for elbow flexors/extensors of 20 participants with tetraplegia [21]. They hypothesized that a positive relationship existed between the angular velocity achieved during the break test and strength measures obtained with the HHD. They con- cluded that when the break test is performed, the velocity applied through the lever arm to "break" the force and initiate an eccentric contraction could lead to variable strength measurements. If muscle spasticity was present, they suggested the make test for improved reliability, because increases in spasticity might require variable increases in force to overcome.

They also found excellent inter- and intrarater reliability between the make and break tests performed by inexperienced examiners on persons with tetraplegia [21]. Additionally, they found no real evidence that either the make or break technique was more favorable than the other. Importantly, they identified statistically that a change of $>3.5 \mathrm{~kg}$ likely represents a true change in strength rather than error. The break/make ratio represents similarities between the two tests, and therefore, the closer to 1 the ratio is, the more similar the results of the two tests. However, similarity should not be interpreted to mean that the two techniques should be used interchangeably or as a normalization approach. Burns and Spanier point out that these ratios varied considerably between individuals.

Hayes et al. evaluated the inter- and intrarater reliability of MMT using an HHD and a spring-scale dynamometer for the shoulder muscles [29]. They found excellent reliability for all movements tested with the HHD between and within raters (0.79-0.92) for elevation, external rotation, and internal rotation. The HHD was the most reliable and discriminatory means to test the rotator cuff musculature. Finally, if more than one dynamometer of the same model is available, the same device should be used for repeat testing of any given individual. Trudelle-Jackson et al. compared two identical HHDs and found a low correlation $($ ICC $=0.58$ ) between the two devices [30].

\section{DYNAMOMETRY FOR GRIP-STRENGTH ASSESSMENT}

Numerous measures of grip strength and methods of interpretation exist. Grip-strength evaluations are used for assessing upper-limb impairment and determining the efficacy of treatment. Grip-strength measurement instruments can be hydraulic, pneumatic, mechanical, or straingauge types. An example of a hydraulic dynamometer is the Jamar ${ }^{\circledR}$ Hydraulic Hand Dynamometer (Sammons Preston, Bolingbrook, Illinois) (Figure 5). It has five widths that allow for different hand sizes. The adjustable 


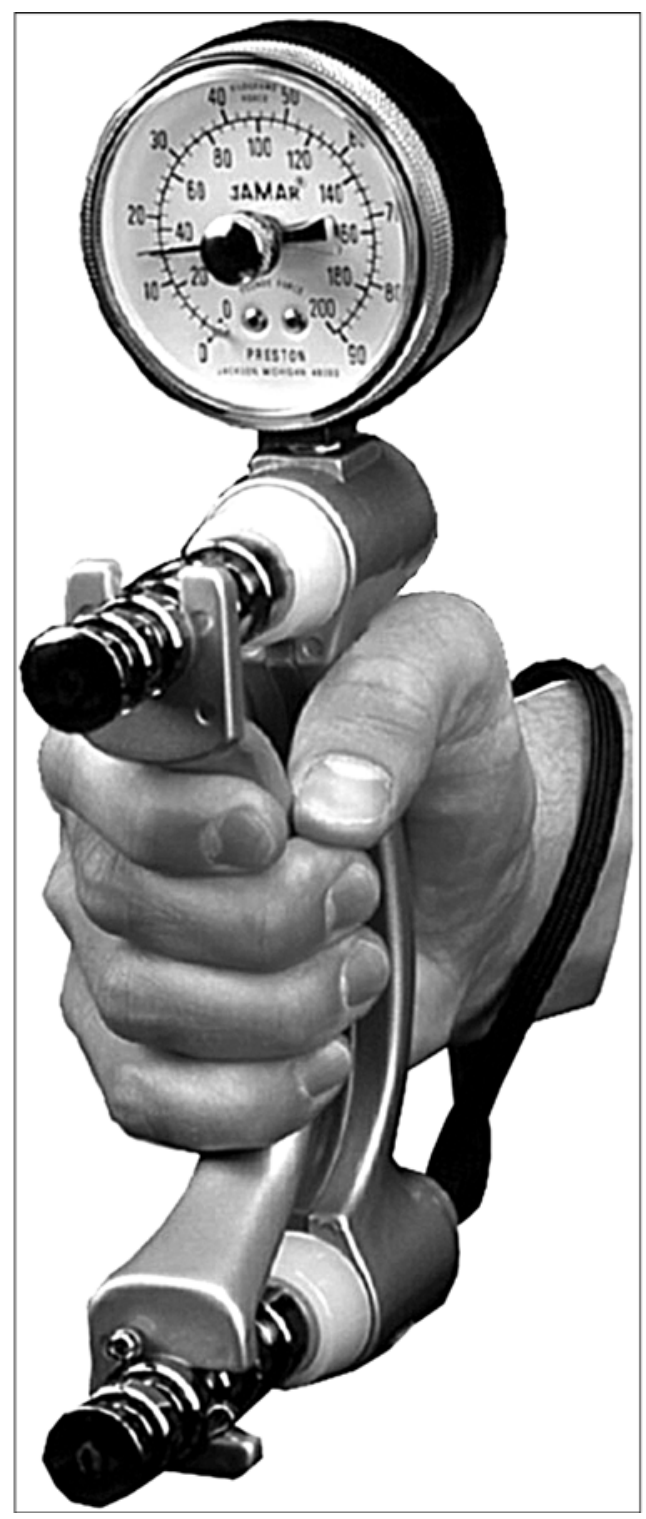

Figure 5.

Jamar $^{\circledR}$ Hydraulic Hand Dynamometer (Model J00105) (Sammons Preston, Bolingbrook, Illinois) has isometric design to ensure accurate, reproducible results and shock-resistant rubber cap that protects stainless-steel gauge. Wrist strap prevents accidental damage if dropped. Figure courtesy of Lafayette Instrument Company.

handle can be placed in five grip positions, from 1.3753.375 in., in 0.5 in. increments. This sealed hydraulic system features a dual-scale readout that displays isometric grip force from 0-200 lb (90 kg). The Jamar dynamometer is the most widely used instrument for measuring grip strength, and many test protocols have been developed because of its widespread use [19].
Pneumatic devices use an air-filled bladder to determine grip strength and are most commonly used in subjects with fragile hands, such as those with rheumatoid arthritis, rather than subjects with SCI. The Tekdyne Hand Dynamometer (Tekdyne Corp, North Wales, Pennsylvania) (Figure 6) is an example a pneumatic dynamometer. These pneumatic devices are often criticized because their pressure measurements (measured in millimeters of mercury or pounds per square inch) are based on the surface area in contact with the device, and the recorded pressure may be larger when a small surface area is tested.

Mechanical devices measure grip strength based on the tension produced by a steel spring. An example of this type of dynamometer is the Smedley dynamometer (Stoelting Company, Wood Dale, Illinois) (Figure 7). It is made from lightweight aluminum and accommodates nearly all hand sizes. Like most handgrip dynamometers, the upper limit of strength measurement is between 220 and $300 \mathrm{lb}$. Finally, strain gauges measure grip strength in Newtons and include digital-pinch analyzers.

The Lafayette Hand Dynamometer features a dualpointer system to retain the maximum effort and $4 \mathrm{~cm}$ of infinite handle adjustment for a comfortable fit (Figure 8). Also, during a grip test, the spring compresses, which provides the handle with realistic dynamic motion. The testing range on a dual scale is $0-100 \mathrm{~kg}$ to the nearest $0.2 \mathrm{~kg}$. The unit contains a custom-twisted spring that can

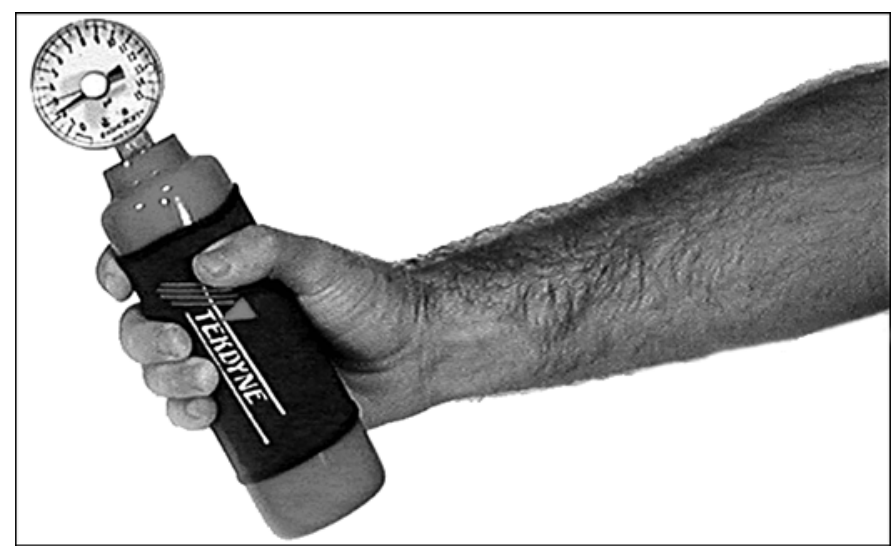

Figure 6.

Tekdyne Hand Dynamometer (Tekdyne, Corp, North Wales, Pennsylvania) measures peak strength through comfortable compression resistance with visible gauge to enable easy reading by examiner and provide feedback to subject. Figure courtesy of Fabrication Enterprises, Inc (C2006). 


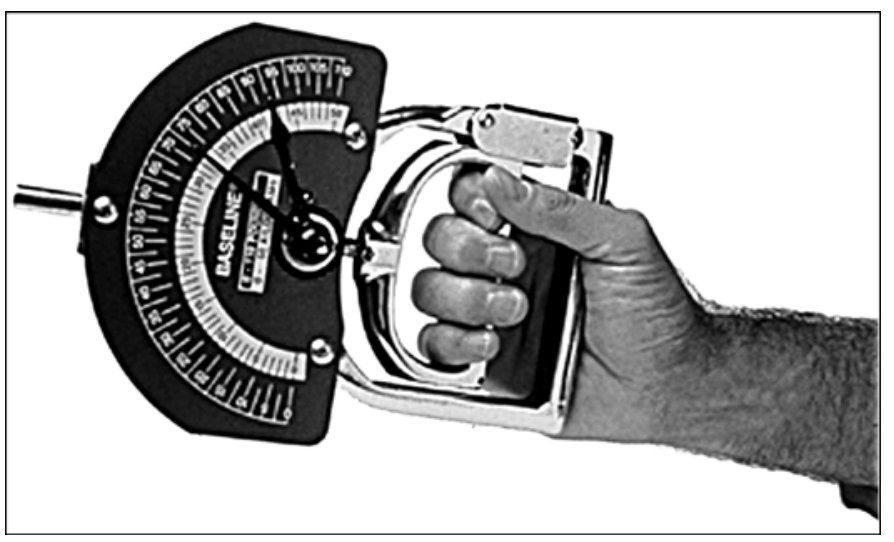

Figure 7.

Smedley (Stoelting Company, Wood Dale, Illinois) spring-type dynamometer measures grip strength. Adjustable handle comfortably accommodates any hand size. Unit is calibrated in both pounds and kilograms. Figure courtesy of NexGen Ergonomics Inc (@2005).

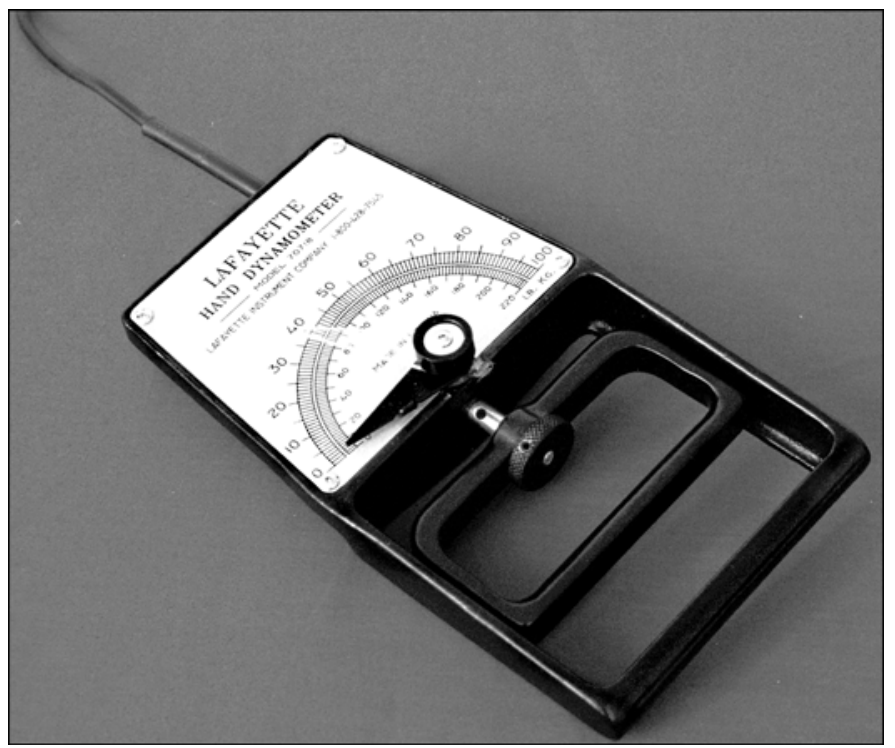

Figure 8.

Lafayette Hand Dynamometer (Lafayette Instrument Company, Lafayette, Indiana).

easily withstand accidental dropping without affecting its accuracy.

Standardized testing position is important for handgrip strength testing. Most researchers and clinicians adopt the standard testing procedure of the American Society of Hand Therapists (ASHT), unless they have a reason to deviate from it. For example, elbow position produces variations in grip strength depending on whether the elbow is bent at $90^{\circ}$ (ASHT position) or straight. Other important factors for consideration when testing grip strength are sex (males have a stronger grip than females), height and weight (the larger the body size, the stronger the grip), dominance (usually a $10 \%$ difference between sides), and level of subject effort [19].

Grip dynamometers may be used to quantify strength changes in persons with lower cervical lesions who retain finger motion or persons with paraplegia with suspected carpel tunnel compression. Grip dynamometers have also been used to measure outcomes in clinical trials of upperlimb tendon transfers [31] and neural prostheses [32]. Handgrip strength has been related to the well-known functional measure, the FIM. The intrinsic muscles of the hand are of special importance in SCI because they are innervated by some of the most caudal segments of the cervical spine. The possibility of peripheral nerve abnormalities and entrapments make intrinsic hand-muscle strength an important metric of neurological decline [33].

\section{RELIABILITY OF GRIP DYNAMOMETERS}

Although most grip-strength instruments have excellent reliability, their results are not interchangeable [19], particularly if their mechanisms are different (e.g., pneumatic vs hydraulic). Even models of the same manufacturer can have different reliability coefficients. The Jamar dynamometer has the highest calibration accuracy of \pm 3 percent when compared with the digital Jamar instrument [34]. The widest hand setting was the most inaccurate with lower loads [35]. The accuracy should be regularly checked based on factory calibration procedures, which is at least once a year or based on the instructions provided by the manufacturer.

Using a dynamometer with normative data from a different instrument is considered incorrect. However, a comparison across instruments on the reliability of the Jamar and Rolyan (Sammons Preston, Bolingbrook, Illinois) hydraulic dynamometers was made. Mathiowetz examined their concurrent validity with known weights as well as their interinstrument reliability and concurrent validity for grip-strength measurements. Mathiowetz concluded that the Jamar and Rolyan dynamometers measure grip strength equivalently and can be used interchangeably and that therapists using the Rolyan dynamometer are justified in using published normative data from the Jamar dynamometer [36]. 


\section{ISOKINETIC DYNAMOMETERS}

For more than 30 years, isokinetic dynamometry has been used in the clinic and in research to evaluate muscle strength. These dynamometers were first validated with known weights [37]. Isokinetic dynamometer reliability studies have been conducted primarily about the knee joint, with less conclusive results at other joints [38]. Isokinetic dynamometry is a method of measuring muscle strength that involves hydraulic or motor-driven devices that impose a constant velocity. The subject is positioned so that the axis of rotation of the mechanical arm is aligned with the axis of rotation of the joint. The subject can either grab a handle or the limb may be strapped onto a mechanical lever arm. This mechanical lever arm measures the subject's torque output when the subject pushes as hard as possible. Muscle strength is defined as the peak torque on the isokinetic peak torque curve, and angular work is calculated as the area under that curve.

Unlike HHDs that measure the force at one particular point in the ROM, isokinetic dynamometers measure torque produced at the anatomical joint throughout the available ROM. Isokinetic dynamometers, such as the KinCom (Chatanooga Corp, Chatanooga, Tennessee), Biodex (Biodex Medical Systems, Inc) (Figure 9), and Lido Active Isokinetic System (Loredan, Inc, Davis, California) measure torque by controlling the velocity of the movement and measuring the force applied via a straingauge force transducer. The KinCom can measure both concentric and eccentric contractions as well as isometric contractions and a special type of isotonic contraction called active assist.

Some disadvantages are associated with isokinetic dynamometers. Cost is a major disadvantage; HHDs can cost between $\$ 400$ and $\$ 1,000$, and isokinetic dynamometers range between $\$ 40,000$ and $\$ 60,000$. Also, when the shoulder joint is tested, maintaining alignment of a fixed axis with the changing shoulder joint axis is difficult. Isokinetic dynamometers are more favorable for the hip, knee, and ankle joints [39]. Another disadvantage is that these devices are large and consequently occupy space in clinical and research departments.

While testing positions are standardized, some testing positions for persons with SCI are cumbersome. Shoulder flexion and abduction strength tests are difficult because the fixed axis becomes misaligned with the changing axis of rotation of the glenohumeral joint. For example, Noreau and Vachon tested shoulder abduction/adduction

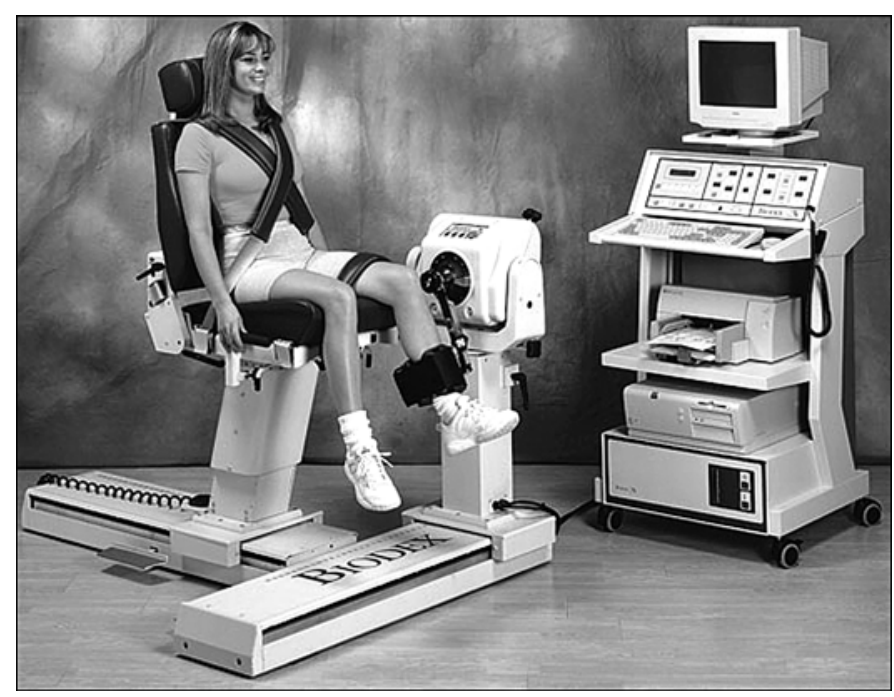

Figure 9.

Biodex System $3^{\mathrm{TM}}$ (Biodex Medical Systems, Inc, Shirley, New York) enables measurement of strength (torque output) throughout range of motion for many muscle groups (shoulder, elbow, and forearm [quadriceps testing shown here]) for concentric and eccentric contractions. Modes for passive range of motion, isometric, and isotonic are available settings. Protections from impact during accelerations/decelerations are provided. Figure courtesy of Biodex Medical Systems, Inc.

with the trunk semireclined at $45^{\circ}$ [17]. This position places the resistance arm in a partially gravity-eliminated position, a position most likely needed for trunk stabilization. Conversely, MMT can be done in sitting (against gravity) or supine (fully gravity eliminated) positions. Therefore, some of the standardized positions required for isokinetic testing may have to be modified for subjects with SCI and may not be comparable with other methods of testing. In some cases, the isokinetic dynamometer cannot be configured properly for clinically relevant muscles and the clinician or researcher may require a distal handgrip device or wrap that applies resistance across two joints, which incorrectly assesses the proximal muscle group.

Souza et al. compared upper-limb strength of 28 persons with paraplegia who were manual wheelchair users with nondisabled control subjects using isokinetic dynamometry [40]. The study was designed to determine whether muscle imbalances existed in individuals with paraplegia who did not participate in sports. Subjects were tested at $60 \%$ with a Biodex system for shoulder abduction/adduction, internal/external rotation, and flexion/extension that was normalized for body weight. No 
significant difference in shoulder strength between persons with paraplegia and nondisabled control subjects was noted. When the subjects with SCI were dichotomized into high- and low-level injury groups, the group with high-level injuries had significantly lower strength measurements than the nondisabled control subjects for shoulder extension. No significant differences were noted in the other planes of motion. The group with low-level injuries had greater shoulder abduction strength than the nondisabled control subjects. The authors correctly pointed out that the limitation of performing isokinetic strength testing of the shoulder muscles while subjects were sitting may have resulted in an overestimation of strength because the trunk muscles aided by generating forces distally. The authors suggest that future studies evaluate strength via torque output in supine positions.

\section{RELIABILITY}

Tests that do not require an examiner to perform the measurement, such as isokinetic dynamometry, yield similar reliability measures as HHDs [5]. Instrumented strength measures produce greater reliability than MMT, generally because of their measurement quality. For example, isokinetic dynamometers or strain gauges produce continuous measurements at a sampling rate significantly high enough to improve variability compared with ordinal measures such as MMT. A device that produces such measures can give output on a continuous basis and enable the detection of improvements in muscle strength when no change may be detected with MMT because a full or half grade would be needed to detect a change. However, these devices are large and cumbersome and sometimes not available to the clinician, either because of the absence of isokinetic dynamometers or strain gauges or limited time available for device calibration and set-up.

The reliability of isokinetic dynamometry depends on the muscles tested. Agre et al. found better reliability with the upper-limb muscles than with the lower-limb muscles [41]. Riddle et al. evaluated patients with cerebral dysfunction and noted greater reliability with the weaker limb [42]. The correlation is generally strong between HHD testing and isokinetic dynamometry. Kilmer et al. compared an HHD with fixed dynamometry (isokinetic) in persons with neuromuscular disorders and nondisabled individuals [22]. Interinstrument reliability was lower for the knee extensors but higher for knee flexors. The results indicated the HHD was reliable for maximum isometric force with the same and different testers when weaker versus stronger (nondisabled) muscles were tested, especially if the same examiner performed serial tests. Tests of powerful proximal muscles in nondisabled individuals may have limited value when performed with an HHD.

Inter- and intraday consistency was evaluated for eccentric contraction of the shoulder abductors at $60 \% \mathrm{~s}$ with the Cybex (Lumex, Ronkonkoma, New York). Six maximum trials were performed on 5 consecutive days, separated by $1-2$ weeks. For the Cybex, intraday correlations were 0.88 to 0.99 and interday correlations were 0.88 to 0.97 . The authors considered variability across trials and concluded that the least variability occurred during the first three trials. The authors concluded that strength changes of $<19$ percent should be considered within the range of measurement error and not clinically significant.

Stratford and Balsor studied the difference in reliability within and between testers for the make and break tests that do not require an examiner (KinCom isometric test) [5]. No difference was noted in the reliability of the make and break tests using the KinCom because of the elimination of the examiner interaction and the consistent rate of resistance to muscle contraction. Moreover, deriving data from a computerized system is more accurate than reading an HHD. More current HHDs have software programs that allow for the download of all test data, which removes the requirement that the examiner see and read a digital screen.

Noreau and Vachon compared methods for testing the upper-limb strength in six muscle groups for 32 persons with SCI (fifth cervical to third lumbar and all ASIA classes) [17]. The authors evaluated three methods for testing upper-limb strength: MMT, HHD, and isokinetic dynamometry. Strength testing was performed at admission and discharge from a rehabilitation hospital. MMT was performed with the participants sitting in a wheelchair. The examiner stabilized the trunk while performing each test. The six muscle groups tested were the shoulder flexors/ extensors, shoulder abductors/adductors, and elbow flexors/extensors bilaterally. The authors used a 10-point MMT scale adapted from the Modified Medical Research Council Scale. For the HHD (Penny and Giles, Gwent, United Kingdom) tests, all participants were tested in the supine position bilaterally in a standardized manner and order with the make test. The same experienced and trained 
examiner performed all tests within and across days. The Cybex II was used for comparison of isokinetic dynamometry with MMT and HHD. Only the stronger side was tested at $60 \%$ in supine and semireclined positions.

The authors concluded that MMT was not sensitive above grade $4 / 5$ or to small or moderate increases in strength. A variable relationship existed between MMT and HHD, especially between admission and discharge because of an increase in muscle strength. The authors also indicated that difficulties may have been present in the sitting MMT procedures, in which trunk stabilization was required. Additionally, because of the overlap between muscle grades across subjects, the correlations decreased [43]. The make test used for the HHD is different from that applied in MMT, which potentially led to lower correlations.

For isokinetic testing, lower correlations were observed compared with the other devices, in particular for shoulder abductors. The lack of proximal stabilization may also have limited the ability of the subject to exert a maximum effort. In certain cases (such as shoulder abduction), the subject had to resist muscle force and gravity for isokinetic testing. Finally, the different testing modes (isometric versus isokinetic) and the different testing positions may have led to lower correlations across devices. This study highlights the importance of comparing devices using the same positions and types of testing (make vs break). The authors concluded by recommending an HHD be used because of its low cost and low assessment time.

\section{DISCUSSION AND CONCLUSIONS}

Standardized strength-testing procedures are important regardless of whether MMT, HHD, handgrip dynamometers, or isokinetic dynamometers are used. MMT is simple, easy to use, and therefore clinically practical. However, the reliability and responsiveness of MMT is uncertain for strength greater than 3/5. HHDs potentially quantify strength numerically by recording force output. Because of the portability of HHDs, they are useful in the clinical/bedside environments for tracking strength changes. The reliability of HHDs is highly dependent on standardized testing procedures, particularly that the examiner have sufficient strength to resist the subject's muscle contraction [44]. The examiner's strength should be greater than that of the specific muscle group being assessed [5]. A drawback of HHDs is that strength is tested at one point in the ROM. Isokinetic dynamometers allow for the measurement of force/torque throughout the available ROM, both concentrically and eccentrically. These devices are not portable and, therefore, not suitable for quick or bedside clinical tests. Measuring strength changes in subjects with SCI can provide evidence of recovery or deterioration of motor output and thereby provide diagnostic information as well as direct the efficiency of rehabilitation interventions.

\section{ACKNOWLEDGMENTS}

This material was based on work supported by the National Institute on Disability and Rehabilitation Research (grants H133N000022 and H133A011107), the Henry H. Kessler Foundation, and the New Jersey Commission on Spinal Cord Research (grant 063054-SCR-EO).

The authors have declared that no competing interests exist.

\section{REFERENCES}

1. Noreau L, Shephard RJ, Simard C, Pare G, Pomerleau P. Relationship of impairment and functional ability to habitual activity and fitness following spinal cord injury. Int J Rehabil Res. 1993;16(4):265-75. [PMID: 8175229]

2. Marciello MA, Herbison GJ, Ditunno JF Jr, Marino RJ, Cohen ME. Wrist strength measured by myometry as an indicator of functional independence. J Neurotrauma. 1995; 12(1):99-106. [PMID: 7783236]

3. Bell GJ, Wenger HA. Physiological adaptations to velocity-controlled resistance training. Sports Med. 1992;13(4): 234-44. [PMID: 1615252]

4. Bohannon RW. Make versus break tests for measuring elbow flexor muscle force with a hand-held dynamometer in patients with stroke. Physiother Can. 1990;42:247-51.

5. Stratford PW, Balsor BE. A comparison of make and break tests using a hand-held dynamometer and the Kin-Com. J Orthop Sports Phys Ther. 1994;19(1):28-32.

[PMID: 8156061]

6. Bohannon RW. Make tests and break tests of elbow flexor muscle strength. Phys Ther. 1988;68(2):193-94. [PMID: 3340656$]$

7. Bohannon RW. Hand-held myometry. J Neurol Neurosurg Psychiatry. 1992;55(10):982. [PMID: 1431978$]$

8. Burns SP, Breuninger A, Kaplan C, Marin H. Hand-held dynamometry in persons with tetraplegia: Comparison of 
make- versus break-testing techniques. Am J Phys Med Rehabil. 2005;84(1):22-29. [PMID: 15632485]

9. Marino RJ, Barros T, Biering-Sorensen F, Burns SP, Donovan WH, Graves DE, Haak M, Hudson LM, Priebe MM. ASIA Neurological standards for neurological classification of spinal cord injury. J Spinal Cord Med. 2003;(26 Suppl 1):S50-56. [PMID: 16296564]

10. Herbison GJ, Isaac Z, Cohen ME, Ditunno JF Jr. Strength post-spinal cord injury: Myometer vs manual muscle test. Spinal Cord. 1996;34(9):543-48. [PMID: 8883189]

11. O’Brien PC, Dyck PJ. Procedures for setting normal values. Neurology. 1995;45(1):17-23. [PMID: 7824110]

12. Frese E, Brown M, Norton BI. Clinical reliability of manual muscle testing. Middle trapezius and gluteus medius muscles. Phys Ther. 1987;67(7):1072-76. [PMID: 3602100]

13. Hislop HJ, Montomery J. Daniels and Worthingham's muscle testing: Techniques of manual examination. 6th ed. Philadelphia (PA): W. B. Saunders Company; 2002.

14. Mendell JR, Florence J. Manual muscle testing. Muscle Nerve. 1990;13 Suppl:S16-20. [PMID: 2233877]

15. Dvir Z. Grade 4 in manual muscle testing: The problem with submaximal strength assessment. Clin Rehabil. 1997;11(1): 36-41. [PMID: 9065358]

16. Beasley WC. Quantitative muscle testing: Principles and applications to research and clinical services. Arch Phys Med Rehabil. 1961;42:398-425. [PMID: 13688259]

17. Noreau L, Vachon J. Comparison of three methods to assess muscular strength in individuals with spinal cord injury. Spinal Cord. 1998;36(10):716-23. [PMID: 9800275]

18. Larsson B, Karlsson S, Eriksson M, Gerdle B. Test-retest reliability of EMG and peak torque during repetitive maximum concentric knee extensions. J Electromyogr Kinesiol. 2003;13(3):281-87. [PMID: 12706607]

19. Innes E. Handgrip strength testing: A review of the literature. Aust Occup Ther J. 1999;46(3):120-40.

20. Andrews AW, Thomas MW, Bohannon RW. Normative values for isometric muscle force measurements obtained with hand-held dynamometers. Phys Ther. 1996;76(3): 248-59. [PMID: 8602410]

21. Burns SP, Spanier DE. Break-technique handheld dynamometry: Relation between angular velocity and strength measurements. Arch Phys Med Rehabil. 2005;86(7):1420-26. [PMID: 16003675]

22. Kilmer DD, McCrory MA, Wright NC, Rosko RA, Kim HR, Aitkens SG. Strength measurement using hand-held dynamometry. Washington (DC): U.S. Department of Education, National Institute on Disability and Rehabilitation Research; 1997.

23. Bankes MJ, Crossman JE, Emery RJ. A standard method of shoulder strength measurement for the Constant score with a spring balance. J Shoulder Elbow Surg. 1998;7(2):116-21. [PMID: 9593088]
24. Leggin BG, Neuman RM, Iannotti JP, Williams GR, Thompson EC. Intrarater and interrater reliability of three isometric dynamometers in assessing shoulder strength. J Shoulder Elbow Surg. 1996;5(1):18-24. [PMID: 8919438]

25. Drolet M, Noreau L, Vachon J, Moffet H. Muscle strength changes as measured by dynamometry following functional rehabilitation in individuals with spinal cord injury. Arch Phys Med Rehabil. 1999;80(7):791-800. [PMID: 10414764]

26. Ditunno JF Jr, Stover SL, Freed MM, Ahn JH. Motor recovery of the upper extremities in traumatic quadriplegia: A multicenter study. Arch Phys Med Rehabil. 1992;73(5): 431-36. [PMID: 1580769]

27. Waters RL, Adkins RH, Yakura JS, Sie I. Motor and sensory recovery following complete tetraplegia. Arch Phys Med Rehabil. 1993;74(3):242-47. [PMID: 8439249]

28. Magnusson SP, Gleim GW, Nicholas JA. Subject variability of shoulder abduction strength testing. Am J Sports Med. 1990;18(4):349-53. [PMID: 2403182]

29. Hayes K, Walton JR, Szomor ZL, Murrell GA. Reliability of 3 methods for assessing shoulder strength. J Shoulder Elbow Surg. 2002;11(1):33-39. [PMID: 11845146]

30. Trudelle-Jackson E, Jackson AW, Frankowski CM, Long KM, Meske NB. Interdevice reliability and validity assessment of the Nicholas Hand-Held Dynamometer. J Orthop Sports Phys Ther. 1994;20(6):302-6. [PMID: 7849750]

31. Mulcahey MJ, Betz RR, Smith BT, Weiss AA. A prospective evaluation of upper extremity tendon transfers in children with cervical spinal cord injury. J Pediatr Orthop. 1999;19(3):319-28. [PMID: 10344314]

32. Haugland M, Sinkjaer T. Interfacing the body's own sensing receptors into neural prosthesis devices. Technol Health Care. 1999;7(6):393-99. [PMID: 10665672]

33. Jacquemin GL, Burns SP, Little JW. Measuring hand intrinsic muscle strength: Normal values and interrater reliability. J Spinal Cord Med. 2004;27(5):460-67. [PMID: 15648801]

34. Mathiowetz V, Weber K, Volland G, Kashman N. Reliability and validity of grip and pinch strength evaluations. J Hand Surg [Am]. 1984;9(2):222-26. [PMID: 6715829]

35. Harkonen R, Harju R, Alarana H. Accuracy of the Jamar dynamometer. J Hand Ther. 1993;6(4):259-62. [PMID: 8124439]

36. Mathiowetz V. Comparison of Rolyan and Jamar dynamometers for measuring grip strength. Occup Ther Int. 2002;9(3):201-9. [PMID: 12374997]

37. Farrell M, Richards JG. Analysis of the reliability and validity of the kinetic communicator exercise device. Med Sci Sports Exerc. 1986;18(1):44-49. [PMID: 3959863]

38. Wessel J, Gray G, Luongo F, Isherwood L. Reliability of work measurements recorded during concentric and eccentric contractions of the knee extensors in healthy subjects. Physiother Can. 1989;41:250-53. 
JRRD, Volume 44, Number 1, 2007

39. Delitto A. Isokinetic dynamometry. Muscle Nerve. 1990;13 Suppl:S53-57. [PMID: 2233888]

40. Souza AL, Boninger ML, Fitzgerald SG, Shimada SD, Cooper RA, Ambrosio F. Upper limb strength in individuals with spinal cord injury who use manual wheelchairs. J Spinal Cord Med. 2005;28(1):26-32. [PMID: 15832901]

41. Agre JC, Magness JL, Hull SZ, Wright KC, Baxter TL, Patterson R, Stradel L. Strength testing with a portable dynamometer: Reliability for upper and lower extremities. Arch Phys Med Rehabil. 1987;68(7):454-58. [PMID: 3606371]

42. Riddle DL, Finucane SD, Rothstein JM, Walker ML. Intrasession and intersession reliability of hand-held dynamometer measurements taken on brain-damaged patients. Phys Ther. 1989;69(3):182-94. [PMID: 2919189]
43. Schwartz S, Cohen ME, Herbison GJ, Shah A. Relationship between two measures of upper extremity strength: Manual muscle test compared to hand-held myometry. Arch Phys Med Rehabil. 1992;73(11):1063-68. [PMID: 1444773]

44. Brinkmann JR. Comparison of a hand-held and fixed dynamometer in measuring strength of patients with neuromuscular disease. J Orthop Sports Phys Ther. 1994;19(2):100-104. [PMID: 8148862]

Submitted for publication November 8, 2005. Accepted in revised form May 15, 2006. 